\title{
EDITORIAL
}

\section{ATS/ERS/WASOG statement on sarcoidosis}

\author{
U. Costabel, G.W. Hunninghake, on behalf of the Sarcoidosis Statement Committee
}

The first international consensus statement on sarcoidosis is currently being copublished in the journals of the American Thoracic Society (ATS) and the World Association for Sarcoidosis and Other Granulomatous Disorders (WASOG) $[1,2]$. This editorial wants to attract the attention of the ERJ readers and European Respiratory Society (ERS) members to this consensus report which is aimed at updating clinicians and scientists about new advances related to sarcoidosis. It is hoped that the document will improve the care of patients with this disorder and trigger new studies to further elucidate the cause and pathogenesis of sarcoidosis. The panel members are experts in the care of patients with sarcoidosis, and the level of evidence for the recommendations made in this statement is largely that of expert opinion developed by consensus. There is little supportive evidence from wellconducted randomized controlled trials.

The panel agreed on the following consensus of the state of the art of the understanding of pathogenesis, diagnosis, and treatment of sarcoidosis:

What we know:

- The clinical features and syndromes

- How to make a diagnosis

- That corticosteroids are an effective short-term therapy

- The incidence and prevalence of the disease

- That some genetic factors alter expression of the disease

- The immunology characteristic of the initial onset of the disease.

What we would like to know:

- If there is a test to predict progression

- If corticosteroids alter the natural history of the disease

- The optimal length of therapy

- If there are less toxic therapies than corticosteroids or cytotoxic agents

- How genetic factors alter expression of the disease

- If genetic factors affect susceptibility to the disease

- The mechanisms of lung injury and fibrosis

- The mechanisms that result in persistent disease

- The cause of sarcoidosis.

The panel was divided into groups responsible for writing specific sections of the statement dealing with definition, history, epidemiology, aetiology and pathogenesis, pathology, clinical presentation and organ involvement, diagnostic approaches, natural history, and treatment of sarcoidosis. The following is a summary of some of the sentinal statements of these sections.

The epidemiology of sarcoidosis remains problematic for several reasons, including: 1) lack of a precise, consistent case definition; 2) variable methods of case ascertainment; 3) variability in disease presentation; 4) lack of

Correspondence: U. Costabel, Abt. Pneumologie/Allergologie, Ruhrlandklinik, Tüschener Weg 40, D-45239 Essen, Germany, Fax: 49201 4334029 sensitive and specific diagnostic tests, resulting in underrecognition and misdiagnosis of the disease; and 5) the paucity of systematic epidemiological investigations of cause. The disease shows a consistent predilection for adults $<40$ yrs of age, peaking in those aged 20-29 yrs. In Scandinavian countries and in Japan, there is a second peak incidence in females $>50$ yrs of age. Significant heterogeneity in prevalence, disease presentation and severity occurs among different ethnic and racial groups. Several studies suggest that sarcoidosis in Afroamericans is more severe, while Caucasians are more likely to present with asymptomatic disease. Overall mortality from sarcoidosis is $1-5 \%$. Intriguing spatial clusters of illness have suggested person-to-person transmission or shared exposure to an environmental agent. Some studies have observed a seasonal clustering of sarcoidosis cases in winter and early spring. There are numerous reports of the familial clustering of sarcoidosis. Human leukocyte antigen (HLA) analyses of affected families suggest that the mode of inheritance of risk for sarcoidosis is to probably polygenic, with the most common genotypes frequencies being class I HLA-A1 and -B8 and class II HLA-DR3. It is likely that genetically predisposed hosts are exposed to antigens that trigger an exaggerated cellular immune response leading to granuloma formation.

Although the aetiology of sarcoidosis remains unknown, there are three different lines of evidence supporting the idea that sarcoidosis results from exposure of genetically susceptible hosts to specific environmental agents: 1) the aforementioned epidemiological studies; 2) the inflammatory response in sarcoidosis, with a pattern of cytokine production in the lungs that is most consistent with a Th1-type immune response triggered by an antigen; and 3) the implications of studies concerning the T-cell receptor (TCR) in patients with sarcoidosis. In regard to the role of environmental agents, the list of possible causative agents

Table 1. - Examples of agents suggested to be involved in the aetiology of sarcoidosis

\begin{tabular}{|c|c|c|}
\hline \multicolumn{3}{|c|}{ Type of agent } \\
\hline Infectious & Inorganic* & Organic \\
\hline $\begin{array}{l}\text { Viruses (herpes, Epstein-Barr, } \\
\text { retrovirus, coxsackie B virus, } \\
\text { cytomegalovirus) }\end{array}$ & Aluminium & Pine tree pollen \\
\hline $\begin{array}{l}\text { Borrelia burgdorferi } \\
\text { Propionibacterium acnes } \\
\text { Mycobacterium tuberculosis } \\
\text { and other mycobacteria } \\
\text { Mycoplasma }\end{array}$ & $\begin{array}{l}\text { Zirconium } \\
\text { Talc }\end{array}$ & Clay \\
\hline
\end{tabular}

*: beryllium, which causes berylliosis and not sarcoidosis, is not included. 
has continuously expanded (table 1). The evidence for an infective aetiology, particularly mycobacterial, is becoming more appealing. Unfortunately, even with the advent of molecular tools, such as the highly sensitive polymerase chain reaction, the debate has not been resolved. Failure to demonstrate mycobacteria may depend on insensitive methods, whereas positive findings may be due to contamination. With respect to the T-cell receptor studies, it is still unclear how helpful they will be in eliciting the aetiology of sarcoidosis.

The immunological abnormalities of the early sarcoid reaction are well known and are characterized by the accumulation of activated T-cells and macrophages at sites of ongoing inflammation, notably in the lung. However, no studies have shown why lung disease persists in some patients but not in others. In addition, no studies have shown how persistent disease results in lung injury and fibrosis. Nevertheless, the immunological pattern of cells in the sarcoid infiltrate suggests that: 1) sarcoid granulomas are formed in response to a persistent and probably poorly degradable antigenic stimulus that induces a local Th1-type T-cell mediated immune response with an oligoclonal pattern; and 2) as a consequence of their chronic stimulation, macrophages release mediators of inflammation, locally leading to accumulation of Th1 cells at sites of ongoing inflammation and contributing to the development of the granuloma structure.

The pathology section describes the morphology of sarcoid granulomas, the location and distribution of the lesion, and the major pathological differential diagnoses. Because important differential diagnoses are infectious diseases, the need for microbiological studies and cultures continues, especially when the patient has fever or when there are necrotic lesions in the biopsy specimens. Special stains for acid-fast bacilli and fungi are justified, especially when there are atypical features for sarcoidosis such as necrosis or an air-space predominance of granulomas.

The clinical presentation and organ involvement is reported in detail, with the lungs being affected in $>90 \%$ of patients with sarcoidosis and the lymphoid system in about one-third. Clinical evidence of heart involvement is seen in $\sim 5 \%$, liver granulomas in as many as $50-80 \%$ of liver biopsy specimens, cutaneous involvement in $\sim 25 \%$, and ocular lesions in $11-83 \%$. Neurosarcoidosis which is clinically recognizable occurs in $<10 \%$ of patients. Symptomatic muscle and bone involvement is rare, whereas joint pains occur in $25-39 \%$. Special situations such as sarcoidosis in children, sarcoidosis in pregnancy, and sarcoidosis in the elderly are also considered.

The diagnosis of sarcoidosis needs a compatible clinical picture, histological demonstration of noncaseating granulomas, and exclusion of other diseases capable of producing a similar histological or clinical picture. The diagnostic work-up for patients with sarcoidosis should attempt to accomplish four goals: 1) provide histological confirmation of the disease; 2) assess the extent and severity of organ involvement; 3 ) assess whether the disease is stable or is likely to progress; and 4) determine whether therapy will benefit the patient. In the presence of a compatible clinical picture, the first step is to choose the site for a proper biopsy. Transbronchial lung biopsy is the recommended procedure in most cases. Its diagnostic yield depends largely on the experience of the operator, ranging from 40 to $>90 \%$. A careful examination of the patient may disclose other possible sites for biopsy, such as skin, lip, or superficial lymph nodes. The problem of a patient without histology needs specific consideration. Some patients ref- use biopsy and in others the pulmonary impairment is too severe for a lung biopsy. Clinical and/or radiological features alone may be diagnostic for patients with Stage I (reliability of $98 \%$ ) or Stage II $(89 \%)$ disease, but are less accurate for patients with Stage III (52\%) or Stage $0(23 \%)$ disease. A patient who presents with a classic Löfgren's syndrome of fever, erythema nodosum, arthralgias, and bilateral hilar lymphadenopathy may not require biopsy proof if resolution of disease is rapid and spontaneous. In some instances, bronchoalveolar lavage and studies on lymphocyte subpopulations are helpful. The appearance of a Panda pattern combined with a Lambda pattern on a total body ${ }^{67} \mathrm{Ga}$ scan may support the diagnosis of sarcoidosis and obviate the need for an invasive diagnostic procedure. A mildly elevated angiotensin-converting enzyme is never diagnostic because elevations may be seen in many diseases.

Once the diagnosis is established, an additional work-up is recommended for all patients (table 2). Lung computed tomography (CT) scans are only indicated for some patients. The usual indications are as follows: 1) atypical clinical and/or chest radiograph findings; 2) detection of complications of the lung disease, such as bronchiectasis, aspergilloma, pulmonary fibrosis, traction emphysema, or a superimposed infection or malignancy; and 3) a normal chest radiograph but a clinical suspicion of the disease. Clinical activity is assessed on the basis of onset, worsening, or persistence of symptoms or signs directly related to sarcoidosis. A long list of markers of activity has been suggested as potential diagnostic aids or indices of "activation". Some of these markers are consistent with the ability of the disease to progress in one organ, but they often do not detect progress in other organs.

The natural history and prognosis of sarcoidosis are highly variable, with a tendency of the disease to wax and wane, either spontaneously, or in response to therapy. Spontaneous remissions occur in nearly two-thirds of patients, but the course is chronic or progressive in $10-30 \%$. Serious extrapulmonary involvement (e.g. cardiac, central nervous system, hepatic) occurs in 4-7\% of patients with sarcoidosis at presentation; the incidence is higher as the disease evolves. Fatalities occur in $1-5 \%$ of patients, typically owing to progressive respiratory insufficiency or central nervous system or myocardial involvement. Several clinical features have been associated with a chronic or progressive course. Adverse prognostic factors include

Table 2. - Recommended initial evaluation of patients with sarcoidosis

Type of evaluation

1. History (occupational and environmental exposure, symptoms)

2. Physical examination

3. Posteroanterior chest radiography

4. Pulmonary function tests: spirometry and $D \mathrm{~L}, \mathrm{CO}$

5. Peripheral blood counts: white blood cells, red blood cells, platelets

6. Serum chemistries: calcium, liver enzymes (alanine aminotransferase, aspartate aminotransferase, alkaline phosphatase), creatinine, BUN

7. Urine analysis

8. ECG

9. Routine ophthalmologic examination

10. Tuberculin skin test

$D \mathrm{~L}, \mathrm{CO}$ : diffusing capacity of the lung for CO; BUN: blood urea nitrogen; ECG: electrocardiogram. 
Table 3. - Alternative therapy for sarcoidosis

\begin{tabular}{|c|c|c|c|c|}
\hline \multirow[b]{2}{*}{ Dosage } & \multicolumn{4}{|c|}{ Drug } \\
\hline & $\begin{array}{l}\text { Methotrexate } \\
10-25 \mathrm{mg} \cdot \mathrm{week}^{-1}\end{array}$ & $\begin{array}{c}\text { Azathioprine } \\
50-200 \mathrm{mg} \cdot \text { day }^{-1}\end{array}$ & $\begin{array}{l}\text { Cyclophosphamide } \\
50-150 \mathrm{mg} \cdot \mathrm{day}^{-1} \text { orally, or } 500-2000 \mathrm{mg} \\
\text { every } 2 \text { weeks intravenously }\end{array}$ & $\begin{array}{l}\text { Hydroxychloroquine } \\
200-400 \mathrm{mg} \cdot \text { day }^{-1}\end{array}$ \\
\hline \multicolumn{5}{|l|}{ Toxicity } \\
\hline Nausea* & 1 & 2 & 3 & 1 \\
\hline Mucosilis* & 2 & 1 & 1 & 0 \\
\hline Haematologic* & 1 & 2 & 3 & 0 \\
\hline Teratogenicity* & 2 & 1 & 3 & 0 \\
\hline Carcinogenic* & 0 & 1 & 3 & 0 \\
\hline Other & Liver, lung & & Bladder & Retinal \\
\hline
\end{tabular}

*: scale: $0=$ none; $1=$ minimal, $2=$ occasional problem, $3=$ significant problem; it may be necessary to adjust the dosage or to use other agents.

lupus pernio, chronic uveitis, age at onset $>40 \mathrm{yrs}$, chronic hypercalcaemia, nephrocalcinosis, black race, progressive pulmonary sarcoidosis, nasal mucosal involvement, cystic bone lesions, neurosarcoidosis, myocardial involvement, and chronic respiratory insufficiency. Numerous studies have affirmed the utility of chest radiographic "stage" as a prognostic guide. Spontaneous remissions occur in 55$90 \%$ of patients with Stage I disease, in $40-70 \%$ of those with Stage II disease, in 10-20\% with Stage III disease, and in $0 \%$ with Stage IV disease.

Longitudinal surveillance of sarcoidosis should be most intensive during the first 2 yrs after presentation, in order to assess prognosis and determine the need (if any) for therapy. For Stage I disease, initial follow-up every 6 months is usually adequate. More frequent evaluations (every 3-6 months) are adviced for Stage II, III or IV sarcoidosis. All patients (irrespective of radiographic stage) should be monitored for a minimum of 3 yrs after therapy is discontinued. Follow-up needs to be more vigilant among patients with corticosteroid-induced remissions, because of the high rate of relapses in this context.

The appropriate treatment of the disorder has not been well defined for all patients. The symptoms and/or findings that necessitate corticosteroid therapy remain controversial. In patients with mild disease, such as skin lesions, anterior uveitis or cough, topical steroid therapy may be all that is necessary. In patients with systemic, symptomatic disease, oral corticosteroids are often employed. Systemic therapy is clearly indicated for cardiac disease, neurological disease, eye disease not responding to topical therapy, and hypercalcaemia. The use of systemic therapy in pulmonary and other extrapulmonary disease is less clear cut, but most physicians feel that progressive symptomatic disease should be treated. A patient with persistent pulmonary infiltrates or progressive loss of lung functions and no symptoms may still require therapy. In patients requiring persistent corticosteroid therapy, antimalarial agents and cytotoxic agents should be considered.

The optimal dose and duration of corticosteroids has not been studied in randomized, prospective trials. Dose and duration of therapy often must be individualized. For pulmonary sarcoidosis, the initial dosage is often $20-40 \mathrm{mg}$. day $^{-1}$ prednisone or its equivalent on alternate days. Higher doses may be necessary for cardiac or neurosarcoidosis. After 1-3 months, the patient should be evaluated for response. Patients failing to respond by 3 months are unlikely to respond to a more protracted course of therapy. At this point, other reasons for failure should be evaluated, such as presence of irreversible, fibrotic disease, noncom- pliance, inadequate dosage, and intrinsic corticosteroid resistance. Among steroid responders, the dose is slowly tapered to 5-10 mg.day ${ }^{-1}$ or an every other day regimen. Treatment should be continued for a minimum of 12 months. Occasionally, a patient with minimal disease of recent onset may respond to therapy over a 3-6 month period. Patients with Löfgren's syndrome do not require therapy with corticosteroids, unless a nonsteroidal agent is not effective. In some patients, recurrent relapses will occur and patients may require long-term, low-dose therapy.

Several cytotoxic agents have been used to treat sarcoidosis. While these agents clearly are of value in selected patients, there are no studies that have clearly delineated when these drugs should be used for therapy. On the basis of safety and efficacy, methotrexate and azathioprine are the preferred agents for most patients. Cyclophosphamide should be reserved for refractory cases (table 3 ).

In patients with fibrotic disease, bronchiectasis often complicates the disease. These patients often require antibiotic therapy. A particular complication of bronchiectasis in sarcoidosis is aspergilloma. Fatal haemoptysis has been noted by many groups. The antifungal agent itraconazole has been used but no clinical trials have demonstrated efficacy. Surgical resection and embolization of the bronchial arteries have been reported as helpful in selected cases. Patients with myalgias and fatigue and those with significant respiratory insufficiency may benefit from pulmonary rehabilitation. Hypoxaemia at rest or with exercise may require supplemental oxygen therapy. Lung and other organ transplantation has been successfully performed for patients with end-stage sarcoidosis.

In summary, the information presented in this statement provides an update of the current knowledge on sarcoidosis. It is hoped that this expert consensus will be of value for clinicians and scientists involved in the management of patients with sarcoidosis.

Committee Members: G.W. Hunninghake (Co-chairman); U. Costabel (Co-chairman); M. Ando; R. Baughman; J-F. Cordier, R. du Bois; A. Eklund; M. Kitaichi; J. Lynch; G. Rizzato; C. Rose; O. Selroos; G. Semenzato; O.P. Sharma.

\section{References}

1. ATS/ERS/WASOG Committee. Statement on Sarcoidosis. Am J Respir Crit Care Med 1999; 160: 736-755.

2. ATS/ERS/WASOG Committee. Statement on Sarcoidosis. Sarcoidosis Vasc Diffuse Lung Dis 1999; 16: 149_ 173. 